\title{
Perspectives on Social Computing
}

\author{
Wendy Kellogg \\ IBM T.J. Watson Research Center, \\ P.O. Box 704, Yorktown Heights, NY 10598 USA \\ wkellogg@us.ibm.com \\ http: //www.research.ibm.com/people/w/wkellogg/index.html
}

\begin{abstract}
Social computing has emerged as a broad area of research in HCI and $\mathrm{CSCW}$, encompassing systems that mediate social information across collectivities such as teams, communities, organizations, cohorts, populations, and markets. Such systems are likely to support and make visible social attributes such as identity, reputation, trust, accountability, presence, social roles, expertise, knowledge, and ownership. Social computing is transforming organizations and societies by creating a pervasive technical infrastructure that includes people, organizations, their relationships and activities as fundamental system components, enabling identity, behavior, social relationships, and experience to be used as resources. In this talk, I argue for a broad definition of social computing, selectively review emerging applications, and discuss current research within and beyond IBM that is driving and is driven by the emerging vision of social computing.
\end{abstract}

Short Bio. Wendy A. Kellogg is one of the founders of the field of social computing, forming the first research group focusing on Social Computing in 1998: the Social Computing Group at IBM's T. J. Watson Research Center. Topics addressed by the group have included social translucence (a conceptual framework pioneered by Erickson and Kellogg), computer-mediated communication, social proxies, the design of social software, knowledge management, awareness systems, enhanced audio conferencing, collaboration and human productivity in high performance computing, social and task visualizations, and most recently, serious games, virtual worlds for business use, and "Enterprise 2.0."

Kellogg's work in human-computer interaction (HCI) over more than two decades has spanned areas including theory, evaluation methods, design, and development. She holds a Ph.D. in Cognitive Psychology from the University of Oregon. She is author and editor of publications in the fields of HCI and CSCW, and currently serves on the editorial board of ACM's Transactions on Computer-Human Interaction. Wendy chaired CHI 2005 Technical Papers, DIS 2000's Technical Program, and the CSCW 2000 and CHI'94 conferences. She chaired Workshops for CHI 2004 and has served numerous times as an associate chair for CHI, CSCW, ECSCW, and DIS. She served on the National Academy of Science's Computer Science and Telecommunications Board and in 2002 was elected ACM Fellow "for contributions to social computing and human-computer interaction." 